\title{
QUÍMICA MEDICINAL: 25 ANOS DE PLANEJAMENTO RACIONAL DE FÁRMACOS
}

\author{
Antonia Tavares do Amaral* \\ Departamento de Química Fundamental, Instituto de Química, Universidade de São Paulo, Avenida Prof. Lineu Prestes, 748, \\ 05508-900 São Paulo - SP \\ Carlos Alberto Montanari \\ Departamento de Química, Universidade Federal de Minas Gerais, CP 702, 31270-901 Belo Horizonte - MG
}

\begin{abstract}
25 YEARS OF MEDICINAL CHEMISTRY IN BRAZIL. The Sociedade Brasileira de Química is commemorating its $25^{\text {th }}$ anniversary, and this paper is intended to draw an overview of the Brazilian Medicinal Chemistry over all these years. In 1977 Brazil had almost no activities at all in the field, albeit many efforts were already on the way for encouraging Brazilian Scientists to enter the area. Among many different endeavours to help medicinal chemists to fulfil their proposals and the establishment of an on-going research with the help of networks, the Sociedade Brasileira de Química created, in 1991, its own Division on Structure and Activity Relationship, which became the Division of Medicinal Chemistry, in 1997.
\end{abstract}

Keywords: Structure Activity Division (DSA); Medicinal Chemistry Division (DMD); 25 $5^{\text {th }}$ anniversary SBQ; drug design.

\section{INTRODUÇÃO}

A Sociedade Brasileira de Química está completando 25 anos de idade. Em conjunturas milenares, trata-se de uma jovem; naquelas referentes à consciência refere-se à maturidade em formação; mas naquelas referentes à ciência trata-se de uma enorme força-tarefa que agrega uma miríade de pesquisas em nível internacional. Acima de tudo a SBQ - embora jovem - tem proporcionado à sua comunidade acesso constante a um celeiro de idéias que, expostas de forma aberta e sem conceitos previamente estabelecidos, tem criado um ambiente científico rigoroso. Saudável, tem efeito multiplicador em todas as áreas da Química. Simultaneamente à sua contribuição agregadora enquanto sociedade científica é preciso enfatizar que a SBQ tem tido papel fundamental na preservação de valores morais, éticos e científicos em toda a comunidade de cientistas, em suas mais distintas áreas de atuação. Por todos esses valores acumulados ao longo destes 25 anos, a SBQ traz este número especial de uma de suas revistas mais importantes, a Química Nova, que estabelece um marco importante para esta celebração. Esta comemoração permite apresentar tanto uma retrospectiva da Química Medicinal no Brasil, seu estado da arte bem como algumas reflexões prospectivas.

\section{NOÇÕES FUNDAMENTAIS SOBRE QUÍMICA MEDICINAL}

Antes de iniciar este relato histórico-cronológico sobre 25 anos de Química Medicinal no Brasil, focando o seu desenvolvimento na SBQ em especial, torna-se importante relembrar o que se entende, nos dias de hoje, por Química Medicinal. Esta, em suas inúmeras atribuições, engloba o planejamento racional de novas substâncias bioativas, envolvendo a síntese ou a modificação molecular de substâncias; o isolamento de princípios ativos naturais (plantas, animais, minerais); a identificação ou elucidação da estrutura; a descrição das moléculas desde a sua constituição atômica (passando por relações entre a estrutura e propriedades) até suas características estruturais quando da(s) interação(ões) com os diferentes sistemas biofases/biológicos; a compreensão em nível molecular de processos bioquímicos/ farmacológicos, toxicológicos e farmacocinéticos. E, finalmente, a

\footnotetext{
*e-mail: atdamara@iq.usp.br
}

proposição e validação de modelos matemáticos através dos estudos de relações entre a estrutura química e a atividade farmacológica e/ ou toxicológica, permitindo então a proposição de novas entidades de interesse. Esta área de conhecimento, utilizando diferentes estratégias metodológicas complementares, é tradicional e reconhecidamente usada no planejamento de fármacos. Esta, no entanto, se estende a diferentes áreas de aplicação, como planejamento e mecanismo de ação de agrotóxicos; previsão da toxicidade de compostos, visando sua aplicação tanto terapêutica quanto ambiental. Cada uma destas etapas, cruciais para o sucesso do planejamento racional de fármacos, é realizada iterativamente. Considerando as disponibilidades tanto de informação e de conhecimento sobre o sistema estudado (composto químico-sistema biológico) bem como de ferramentas teóricas e de recursos experimentais, poder-se-á atingir os vários níveis de desenvolvimento, alcançados em cada uma das etapas. Estas etapas, podem ser mais bem visualizadas no esquema apresentado na Figura 1. Deste modo, o desenvolvimento e sucesso em química medicinal requerem o conhecimento e aplicação de estratégias metodológicas híbridas, no centro de um grande espaço inter-, transe multidisciplinar fundamentadas por conhecimentos, por exemplo, em biofísica, biologia molecular, bioquímica, clínica médica, estatística, físico-química, fisiologia, matemática, neurobiologia, patologia, química biológica, química inorgânica, química orgânica e química quântica.

Como consequiência, o planejamento de moléculas bioativas e, mais ainda, relevantes para o país, só poderia ocorrer em um sistema integrado e iterativo de redes, em cooperações mútuas, semelhantes a um equilíbrio dinâmico. Este talvez tenha sido um dos problemas enfrentados nos idos da década de 1970, quando a capacidade de agrupar era muito mais eficiente em movimentos políticos, não em movimentos científicos. Atualmente, essa perspectiva mudou. Entretanto, muito há que se fazer para que a iteratividade da química medicinal the valha amplos frutos.

Nesse contexto, a formação de recursos humanos, com este perfil multidisciplinar e com alto valor de competência, se tornou fundamental para o desenvolvimento desta área de conhecimento em nosso meio.

Para, adicionalmente, se atingir a iteratividade necessária estabeleceu-se um importante marco em 1991, quando foi criada a Divisão de Estrutura Química e Atividade Biológica, DSA, da SBQ, que seis anos depois viria a se tornar a Divisão de Química Medicinal, DMD. 
Várias inserções durante reuniões da SBPC, da SBBq e também em reuniões da Seção de Química Biológica da SBQ, foram realizadas pelo grupo de pesquisadores brasileiros em físico-química orgânica, interessados em estender os conhecimentos já consagrados dessa área para o entendimento dos sistemas biológicos. Apesar desses esforços, somente com o advento da DSA é que se materializou, pela primeira vez de forma conjunta, a união entre as duas áreas: a físico-química orgânica e a biológica.

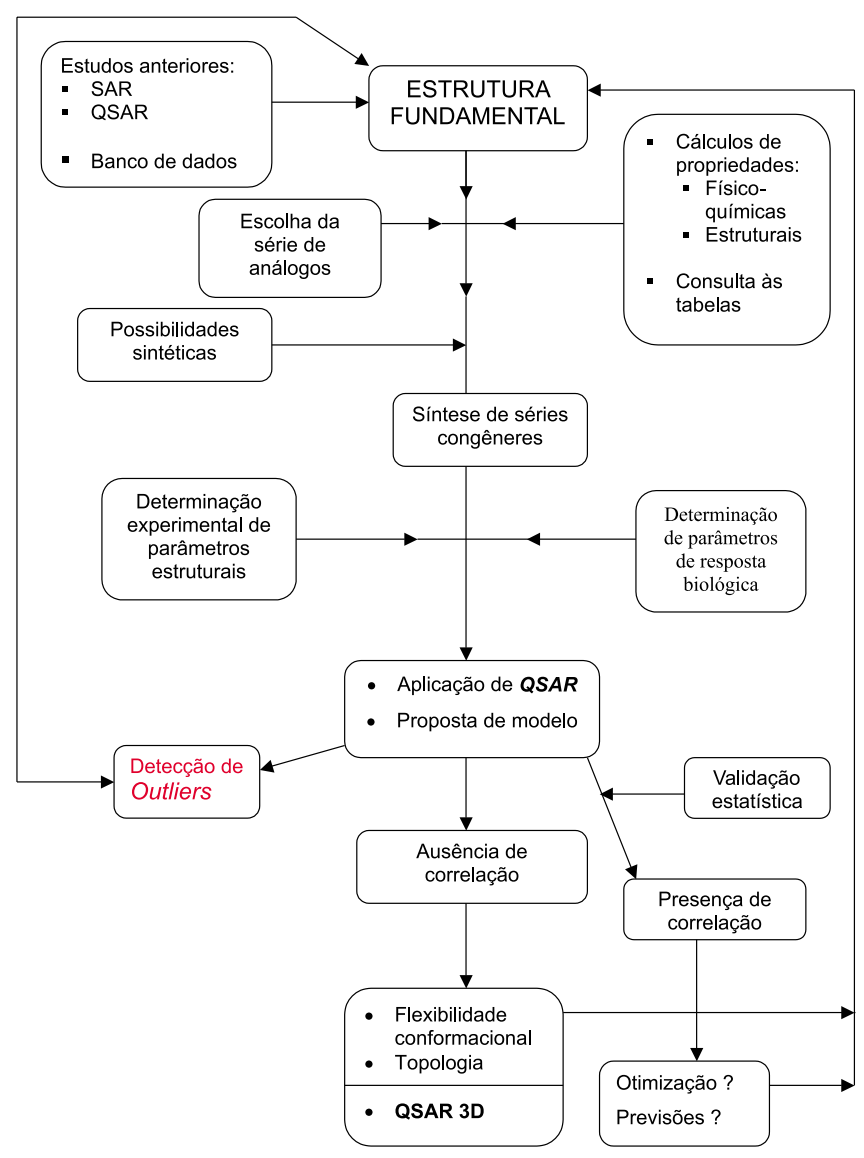

Figura 1. Química Medicinal no planejamento de compostos biologicamente ativos

Com a criação da DSA, tornou-se possível, talvez pela primeira vez de forma sistemática, a união de vários pesquisadores de diferentes especialidades dentro de uma mesma reunião, com o objetivo de demonstrarem, individualmente, suas experiências e como usá-las dentro do contexto multidisciplinar da DSA, particularmente em planejamento de moléculas bioativas. A DSA possibilitou, então, a sistematização e gerenciamento dessas diferentes abordagens. A introdução dessa forma de pensar despertou enorme interesse entre os pesquisadores mas, ao mesmo tempo, causou certa perplexidade ao constatarse que a união de diferentes especialidades poderia contribuir para a compreensão do sistema biológico, através de modificações da estrutura molecular. Felizmente, a euforia demonstrada pela possibilidade de se modificar a estrutura de tal forma que o sistema biológico pudesse responder a essa modificação, fez com que esses pesquisadores engrossassem sua participação nas reuniões anuais até que em 1997 a DSA foi renomeada para a DMD. Nesse momento, como já havia previsão da assembléia divisional, houve um aumento significativo do número de participantes ativos sócios da DMD. Em termos numéri$\cos$, durante as reuniões da DSA houve contribuição científica de cer- ca de 30 trabalhos/ano. Esse número, já no primeiro ano da DMD aumentou para cerca de 80/ano. Por ocasião das realizações dos dois primeiros workshops sobre Relações Quantitativas entre a Estrutura Química e a Atividade Biológica, QSAR, (1991 e 1994), no IQUSP, SP, a audiência foi constituída de pesquisadores de diferentes partes do Brasil em cerca de 100 participantes cada uma delas. Em 2001, durante a realização do $3^{\text {rd }}$ Workshop on Chemical Structure and Biological Activity. Perspectives in QSAR e também do $1^{\text {st }}$ Brazilian Symposium on Medicinal Chemistry, BRAZMEDCHEM, o número de participantes aumentou para cerca de 130 cada, o que demonstra, claramente, a evolução do interesse pela área em poucos anos de sua existência enquanto sociedade organizada para o estudo das atividades inerentes à química medicinal. Observou-se claramente não apenas o aumento de participantes, mas também a qualidade dos trabalhos foi mantida e a cooperação entre os diferentes grupos de pesquisa passou a ser evidenciada. Essa mudança é importante do ponto de vista de agregação da química medicinal e estabelece-se por ser fundamental à iteratividade acima descrita.

\section{CENÁRIO CIENTÍFICO E TECNOLÓGICO NO PAÍS ANTES DE 1991}

Segundo comunicação pessoal do professor Toshio Fujita, o pesquisador pioneiro Corwin Hansch visitou o Brasil já nos idos de 1966, durante o terceiro Simpósio Internacional de Farmacologia, na Universidade de São Paulo, quando ministrou palestra sobre relações entre estrutura química e atividade farmacológica, mostrando aquilo que viria a ser conhecido como a abordagem de Hansch-Fujita. Sua vinda ao Brasil ocorreu por convite do saudoso professor Rocha e Silva, um dos organizadores da fase brasileira do evento ${ }^{1}$.

Em 1964, o professor Corwin Hansch publicou o primeiro artigo versando sobre relações quantitativas entre a estrutura química e a atividade farmacológica ${ }^{2}$ e, anos depois o Brasil entrava para o cenário internacional com algumas publicações históricas dos professores Andrejus Korolkolvas e Ivan da Rocha Pitta ${ }^{3-5}$.

Também de forma pioneira, o saudoso professor Andrejus Korolkovas escreveu e traduziu seu livro Medicinal Chemistry ${ }^{6-10}$ para o português. Com isso, uma verdadeira escola se formou, principalmente em meio farmacêutico, onde a disciplina de farmacologia/química farmacêutica é ministrada. A produção literária na área continuou evoluindo e atualmente culminou com a publicação de um novo livro sobre a natureza atual da química medicinal ${ }^{11}$.

\section{CENÁRIO CIENTÍFICO E TECNOLÓGICO NO PAÍS DEPOIS DE 1991}

Uma das primeiras tentativas de sistematização e divulgação das atividades inerentes à química medicinal, com significativo efeito multiplicador, ocorreu nos idos de 1988-89 quando se iniciou um intercâmbio de pesquisadores do Brasil e do exterior. Especificamente na área de QSAR (relações quantitativas entre a estrutura química e a atividade biológica, uma das áreas de atuação da química medicinal) esta se deu com a participação de diversos pesquisadores estrangeiros, mas que contou com a dinâmica contribuição do professor Hugo Kubinyi, do Instituto de Química Farmacêutica, Universidade de Heidelberg, e da BASF Ludwigshafen, Alemanha.

Durante os primeiros cinco anos, as metodologias utilizadas em QSAR, suas potencialidades e limitações foram divulgadas, em diferentes regiões do Brasil, por meio, principalmente, de atividades no âmbito da pós-graduação e pesquisa. Pretendeu-se, desta forma, tanto divulgar a área quanto despertar o interesse para a realização de trabalhos de pesquisa com efetiva colaboração.

A segunda etapa compreendeu o desenvolvimento de projetos de 
pesquisa, efetuados agora em colaboração com outros grupos, de Institutos de Pesquisa e de Universidades, envolvendo grupos de pesquisa no Brasil. Estes foram e, estão sendo factíveis, através de financiamentos, na grande maioria das vezes provenientes das diferentes agências de fomento, para com bolsas de doutorado, mestrado e iniciação científica. Entre os projetos financiados, muitos eram de caráter individual e alguns em colaboração.

Como resultado deste intercâmbio, realizou-se no IQUSP, São Paulo dois workshops sobre Chemical Structure and Biological Activity, respectivamente em 1991 e 1994. O grande interesse despertado se avalia pelo elevado número de participantes, apresentando painéis (mais de 100, em cada um deles), vindos de várias partes do Brasil, e América do Sul.

$\mathrm{O}$ efeito multiplicador destes dois eventos foi significativo e determinante para as ações arroladas como seguem:

1. surgimento da Divisão Estrutura Química e Atividade Biológica, (DSA), na SBQ, precursora da Divisão de Química Medicinal, (DMD), formada em 1998;

2. formação de núcleos de pesquisa (envolvendo pesquisadores e alunos de pós-graduação) na área de QSAR/QSAR$3 \mathrm{D}$, em instituições de ensino e pesquisa e em institutos de pesquisa;

3. realização de cursos de pós-graduação;

4. realização de visitas de pesquisadores brasileiros provenientes dos vários núcleos de pesquisa aos laboratórios especializados no exterior;

5. crescimento expressivo da participação de pesquisadores brasileiros em congressos da área no exterior;

6. inserção de pesquisadores brasileiros junto à comunidade científica internacional.

Paralelamente, é notória, entre outras, a importância da Escola de Química Farmacêutica no Rio de Janeiro, criada em 1995, que tem contribuído para a formação de recursos humanos, principalmente em níveis de graduação.

Todos esses esforços resultaram em um crescimento significativo da comunidade científica brasileira, que culminou com a realização do $3^{\text {rd }}$ Workshop on Chemical Structure and Biological Activity. Perspectives in QSAR e do $1^{\text {st }}$ Brazilian Symposium on Medicinal Chemistry, BRAZMEDCHEM, ambos em 2001, com cerca de 130 participantes. Isto reflete o nível de atuação dos pesquisadores que têm atuado na área de QSAR e mais amplamente em Química Medicinal.

As Figuras 2 a 5 mostram as características desses dois eventos em termos da origem das atividades nas mais diferentes áreas da química medicinal, bem como a qualificação de seus participantes.

Uma busca exploratória foi realizada no Índice de Citações da FAPESP/CAPES ${ }^{12}$, com o objetivo de identificar as publicações dos diferentes grupos de pesquisa que atuam em química medicinal no Brasil. Esta busca, deu-se pelo uso de algumas palavras-chave e teve o único e precípuo interesse em mostrar a evolução da área desde 1977. Portanto, não se trata de uma busca exaustiva. Apenas por brevidade inerente, as palavras escolhidas foram: QUÍMICA MEDICINAL ${ }^{13-22}$, QSAR $^{14,23-62}$ e PLANEJAMENTO DE FÁRMACOS ${ }^{14,18,21,44,45,56,57,59,}$ ${ }^{63-79}$. As Figuras 6 a 8 mostram os respectivos gráficos.

Na última década, um dos maiores avanços da química medicinal ocorreu, certamente, na área de química combinatória. Embora ainda modestos numerologicamente, são 10 trabalhos publicados no período de 1998 a 2001, sendo 5 deles somente no ano de 2001. Foram 82 artigos usando as palavras-chave lipofilia ou hidrofobia no período de 1982 a 2001, enquanto que outros 141 artigos estavam relacionados aos termos quimiométricos PCA, PLS, SIMCA, KNN ou MRA publicados entre 1979 e 2001, sendo que destes últimos 25 ocorreram somente em 2001 e 16 em 2000.

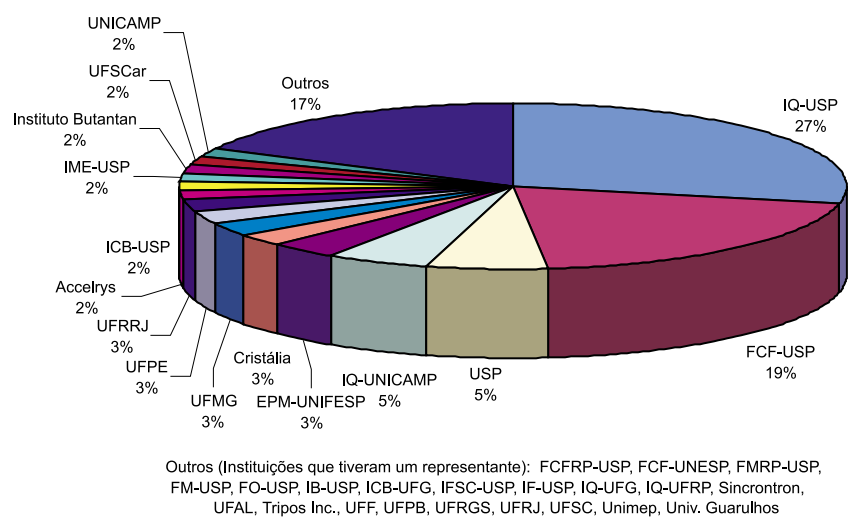

Figura 2. Número de participantes por instituição, durante o $3^{\text {rd }}$ Workshop on QSAR, IQ/USP, 8-11 de novembro de 2001

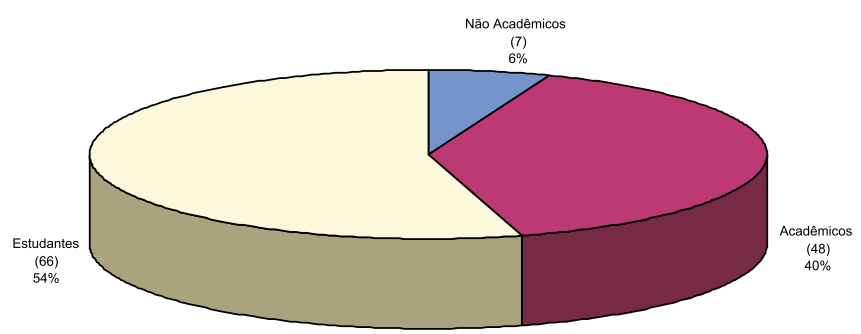

Figura 3. Qualificação dos participantes do $3^{\text {rd }}$ Workshop on QSAR

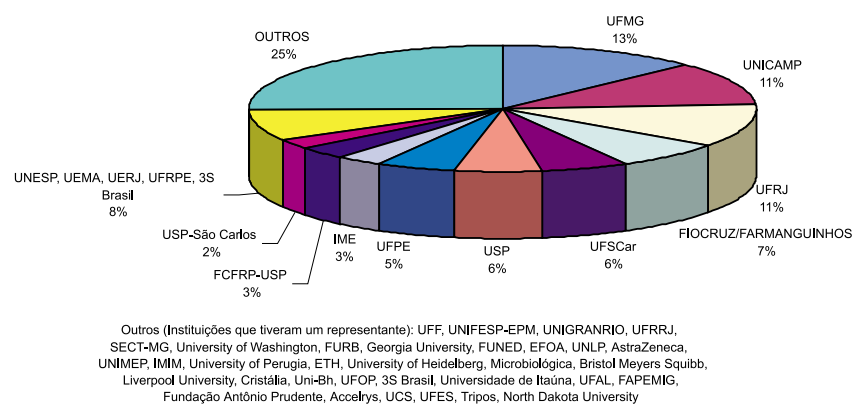

Figura 4. Número de participantes por instituição, durante o $1^{\text {st }}$ Brazilian Symposium on Medicinal Chemistry, BRAZMEDCHEM, 11-16 de novembro de 2001

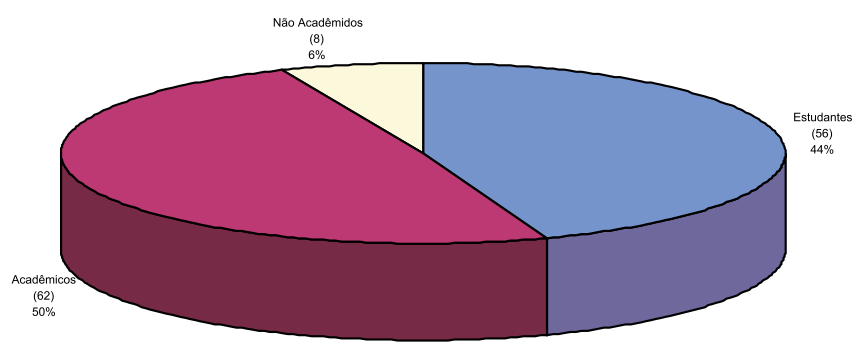

Figura 5. Qualificação dos participantes do BRAZMEDCHEM 


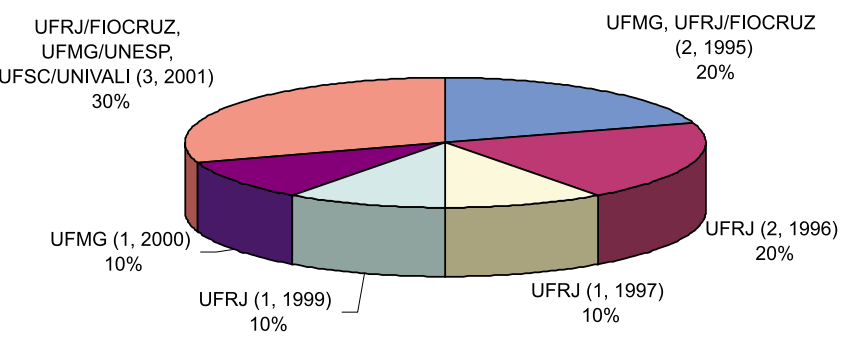

Figura 6. Participação brasileira no cenário internacional com contribuições em QUÍMICA MEDICINAL

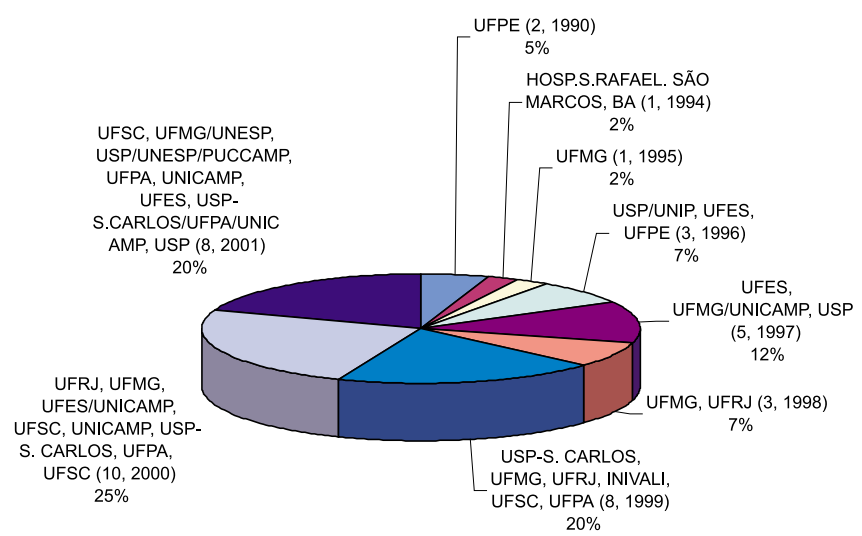

Figura 7. Participação brasileira no cenário internacional com contribuições em QSAR

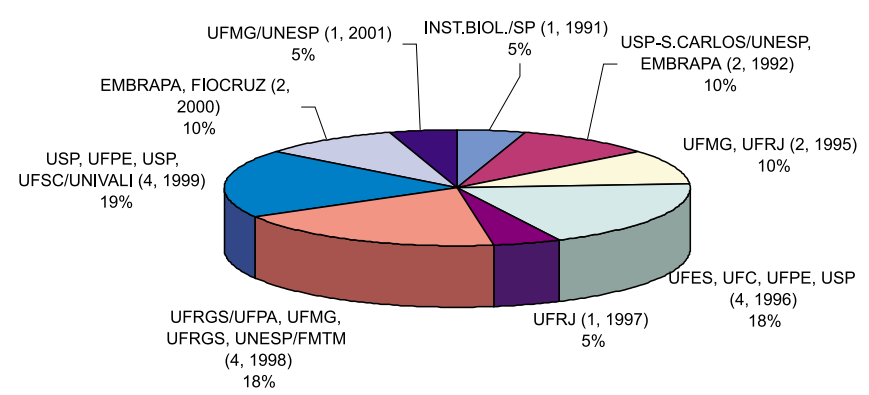

Figura 8. Participação brasileira no cenário internacional com contribuições em PLANEJAMENTO DE FÁRMACOS

Usando as palavras-chave química medicinal, QSAR e planejamento de fármacos uma busca no Diretório de Grupos de Pesquisa do CNPq, versão 1, 1993, foram obtidos 43 grupos. As áreas de ciências exatas, saúde, biológicas e agrárias estavam contempladas. Com o unitermo química medicinal, foram 3 grupos; 2 para QSAR e 1 para planejamento de fármacos. Dez citações para PCA e 4 para quimiometria. Em busca conceitual na versão 2, 1995, do mesmo diretório, foram 2 grupos relacionados à química medicinal; 123 documentos para QSAR (9 para busca simples); 3 grupos e 2997 documentos para planejamento de fármacos (853 em busca simples). Na versão 3 de 1997, foram 10 grupos e 3336 documentos para química medicinal em busca conceitual e 1485 em busca simples; 70 documentos para QSAR (12 em busca simples); 2 grupos e 2238 documentos em planejamento de fármacos ( 930 em busca simples). Nessa ver- são, a busca por linhas de pesquisa foi incluída e os resultados foram os seguintes: química medicinal, 12 grupos e 9127 (3052) documentos; QSAR, 856 (18); planejamento de fármacos, 5629 (1115) documentos. A versão atual 4.1 apresentou os seguintes resultados em busca exata dos termos: química medicinal, 34 grupos de pesquisa; QSAR, 77 e planejamento de fármacos, 15 . Curiosamente, as linhas de pesquisa em química medicinal somaram apenas 21!; QSAR, 16. É importante ressaltar que grupos trabalhando em biologia estrutural, como por exemplo em cristalografia estrutural, aparecem com vínculos específicos a esses estudos. Isso demonstra, claramente, a evolução da natureza pluridisciplinar da área; e, planejamento de fármacos, 2, que muda para 21 quando ambas as palavras são buscadas.

O Instituto Nacional de Propriedade Intelectual, INPI, (http:// www.inpi.gov.br/), tinha, em 6 de janeiro de 2002, 836 registros de patentes em medicamentos, 6 em fármacos, 355 em drogas. A Figura 9 exemplifica as patentes de fármacos na área parasitológica, registradas no INPI.

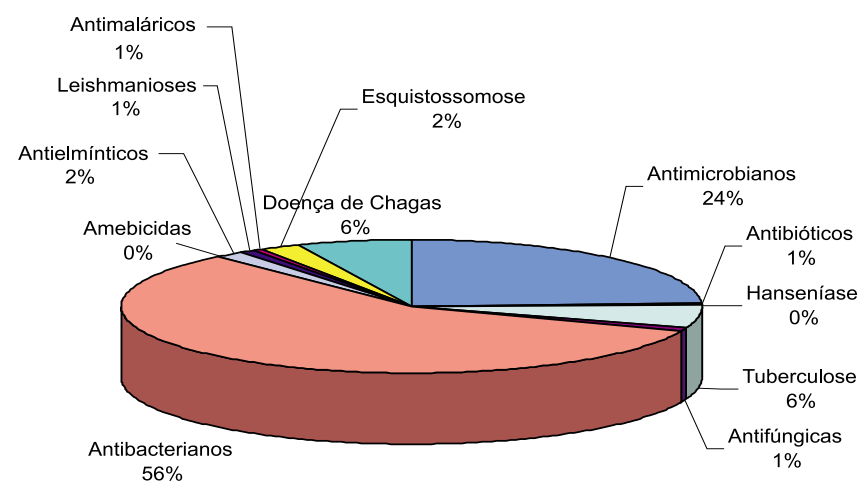

Figura 9. Classes de fármacos de ação contra organismos parasitas, registradas no INPI

\section{Os desafios da química medicinal brasileira}

Dois sustentáculos importantes precisam ser delineados: o referencial brasileiro para a química medicinal e sua inserção no cenário internacional.

Inicialmente e independente do estabelecimento das referências de trabalho, é necessário enfatizar que as abordagens metodológicas empregadas e inerentes ao estudo da interação composto químicosistema biológico precisarão ser de domínio comum.

Os principais desafios que a química medicinal poderá experimentar nos anos vindouros podem ser referenciados através da química do século XX: não basta catalogar informações, é preciso saber como gerenciá-las. Nesse contexto o manuseio de um grande número de dados apresenta especial interesse, que irão permitir:

- apontar (quantitativamente) as características estruturais importantes para a atividade biológica e com elevado poder de predição, deste modo, novas "estruturas-candidato" podem ser propostas;

- avaliar a similaridade/dissimilaridade estrutural, incluindose estruturas flexíveis e coleções combinatórias;

- propor métodos experimentais e de simulação da permeabilidade de membranas, partição e solubilidade;

- propor métodos experimentais que possam ser extrapolados, para um grande número de moléculas ( > >1000) e com alto poder de previsão;

- realizar ensaio virtual (virtual screening);

- construir coleções dirigidas de compostos químicos (target libraries); 
- identificar e propor novas estruturas mínimas essenciais para a atividade biológica de interesse (needle structures);

- reduzir dados sem perda de informação;

- avaliar aspectos metodológicos e teóricos na predição da interação ligante-proteína;

- derivar modelos de QSAR, com o conhecimento da estrutura da proteína, nos dias de hoje, cada vez mais comuns;

- determinar série representativa da estrutura de proteínas que cobre proteínas biologicamente relevantes. Identidade e similaridade na sequiência de proteínas;

- propor métodos de predição da função da proteína a partir da estrutura 3D, identificando regiões de interação, através de banco de dados ou data mining;

- detectar cavidades na proteína importantes para a atividade. Banco de dados de cavidades para planejamento de fármacos pode ser um novo alvo, pois fornece informação sobre a for$\mathrm{ma}$;

- propor novas abordagens em Quimiometria;

- aplicar métodos quimiométricos em ADMET;

- propor estratégias de superposição e alinhamento, incluindo estruturas flexíveis;

- $\quad$ sugerir novas compostos ativos a partir de structure-based libraries;

- construção e validação de grande banco de dados e bibliotecas combinatórias (coleções);

- utilizar data mining no planejamento de fármacos;

- utilizar métodos computacionais em terapia gênica;

- estender os conhecimentos da Química Medicinal acadêmica para a indústria e vice-versa;

- estender todas as estratégias metodológicas para outras áreas de aplicação da química medicinal, que tradicionalmente se utiliza apenas a medicamentos como, por exemplo, meio-ambiente e toxicologia, agroquímicos;

- definir alvos relevantes para as doenças negligenciadas e de interesse para o Brasil, em especial;

- construir bancos de dados taxio- e quimiotaxonômicos em produtos naturais, com informações biológicas relevantes, como fonte de matéria prima para a química medicinal. Esses bancos de dados precisarão ser de domínio público.

Não menos importante mas, ao contrário, essencial para que todas essas estratégias sejam factíveis, é preciso que as agências de fomento e também a comunidade e qualquer outra fonte de financiamento da pesquisa no Brasil, entendam e reconheçam que a química medicinal, DMD, é uma das áreas de caráter essencial para a ciência brasileira, bem como sua inserção no contexto internacional. E, não simplesmente um repositório de substâncias químicas bioativas que jamais se tornarão fármacos, herbicidas, pesticidas, etc...

\section{CONCLUSÃO}

Os desafios da química medicinal no Brasil somente poderão ser enfrentados e, soluções apresentadas com o estabelecimento de cooperações mútuas em redes. Há um entendimento de que as redes sejam estabelecidas de tal forma que os nós se constituam de pequenos aglomerados de especialistas (tipicamente, entre 6 e 10 membros), associados a outros nós de configuração similar mas de diferentes especialidades. Com isso, a diversidade de atuação das especialidades dar-se-á de forma a atender as diferentes interfaces necessárias para a composição da química medicinal. O sucesso depende do estabelecimento dos referenciais brasileiros. Dessa forma, grupos de pesquisadores e/ou pequenas empresas serão constituídas com base de capital de empreendimento de risco, financiados por agentes financeiros de capital privado e/ou público, ou pelas próprias indústrias. Esses núcleos assim organizados terão a incumbência de atuar nas mais diferentes áreas prioritárias, com a finalidade de desenvolver/intermediar tecnologia de curto e longo prazos, minimizando as diferenças eventualmente existentes entre todos os nós da rede, incluídas a indústria e a academia. Destarte, doenças consideradas negligenciadas em países em desenvolvimento, como o Brasil, poderão ser estudadas, mesmo que seja apenas em seus estágios iniciais de planejamento. A AIDS e a tuberculose constituem bons exemplos da importância que o país tem em estudar suas etiologias.

Os principais estágios que devem ser seguidos no descobrimento de novas substâncias químicas iniciam-se com a obtenção da estrutura tridimensional do alvo. Independente de ser esse alvo (ou alvos) novo ou não, modelos comparativos de proteínas relacionadas (pertencentes à mesma família) são essenciais para a identificação das classes de moléculas candidatas. Após a sua identificação, uma coleção virtual de compostos, em geral de domínio público, poderá ser avaliada frente a esses alvos e/ou modelos. As melhores moléculas candidatas serão, então, selecionadas e testadas contra o alvo especificado inicialmente, racionalizando as características estruturais mínimas que descrevem a afinidade.

As moléculas candidatas poderão ser estruturalmente relacionadas ou não, dependendo da seleção prévia com base no alvo e nos modelos. Se forem distintas, para cada uma delas uma coleção de compostos será planejada com base definida em termos da natureza da interação com o alvo. A coleção poderá ser testada virtualmente contra o alvo ou, dependendo da capacidade de ensaio e experimentalmente contra o alvo. Desse ensaio, evolverão, muito provavelmente, várias moléculas candidatas. A transformação dessas moléculas candidatas em substâncias matrizes far-se-á, essencialmente, por compostos similares mais potentes. Os estudos de otimização por QSAR e de ADMET (absorção, distribuição, metabolismo, excreção e toxidez) serão então realizados e validados. Somente as estruturas das moléculas otimizadas prosseguirão para os estudos in vivo e então selecionadas para os ensaios pré-clínicos e clínicos. A patente faz-se mister neste momento e a busca pela cooperação da indústria, fundamental.

\section{REFERÊNCIAS}

1. Hansch, C.; The Use of Substituent Constants in Structure -Activity Studies: em International Pharmacological Meeting, 3, 1966, São Paulo, Proceedings. Oxford: Pergamon Press 1968, 7, 141.

2. Hansch, C.; Fujita, T.; J. Am. Chem. Soc. 1964, 86, 1616.

3. Dantas, M.F.; Pitta, I. R.; Galdino, S.L.; Korolkovas. A.; Rev. Farm. Bioquím. 1982, 18, 99.

4. Melo, S.J.; Galdino, S.L.; Pitta, I. R.; Campos, M.A.; A. Korolkovas; Quim. Nova 1985, 8, 13.

5. Galdino, S.L.; Pitta, I.R.; Luu-Duc., C.; Il Farmaco 1986, 41, 59.

6. Korolkovas, A.; Burckhalter, J.H.; Química Farmacêutica, Guanabara Dois: São Paulo, 1982.

7. Korolkovas, A.; Análise Farmacêutica, Guanabara Dois: São Paulo, 1984

8. Korolkovas, A.; Dicionário Terapêutico, Guanabara Koogan: Rio de Janeiro, 1996.

9. Korolkovas, A.; Essentials of Medicinal Chemistry, $2^{\text {nd }}$ ed., WileyInterscience: New York, 1988.

10. Korolkovas, A.; Fundamentos de Farmacologia Molecular: Bases para o Planejamento de Fármacos, EDART/EDUSP: São Paulo, 1974.

11. Barreiro, E. J.; Mansur, C. A.; Química Medicinal: As Bases Moleculares da Ação dos Fármacos, ArtMed Editora Ltda: Porto Alegre, 2001.

12. http://webofscience.fapesp.br, acessada em Janeiro 2002.

13. De Moura, K. C. G.; Emery, F. S.; Neves-Pinto, C.; Pinto, M. D. F. R.; Dantas, A. P.; Salomão, K.; de Castro, S.L.; Pinto, A. V.; J. Braz. Chem. Soc. 2001, 12, 325.

14. Montanari, C. A.; Bolzani, V. da S.; Quim. Nova 2001, 24, 105.

15. Yunes, R. A.; Pedrosa, R. C.; Cechinel Filho, V.; Quim. Nova 2001, 24, 147.

16. Montanari, C. A.; Quim. Nova 2000, 23, 134. 
17. Filgueiras, C. A. L.; Quim. Nova 1999, 22, 614.

18. Barreiro, E. J.; Rodrigues, C. R.; Albuquerque, M. G.; Sant'Anna, C. M. R. de; Alencastro, R. B. de; Quim. Nova 1997, 20, 300.

19. Barreiro, E. J.; Silva, J. F. M. da; Fraga, C. A. M.; Quim. Nova 1996, 19, 641.

20. Fraga, C. A. M.; Barreiro, E. J.; Quim. Nova 1996, 19, 182.

21. Freitas, A. C. C.; Barreiro, E. J.; Pereira, A. D. L.; Pereira, E. F. R.; Pereira, N. A.; Quim. Nova 1995, 18, 138.

22. Montanari, C. A.; Quim. Nova 1995, 18, 5.

23. Pires, J. T.; Saito, C.; Gomes, S. L.; Giesbrecht, A. M.; Amaral, A. T.-do.; J. Med. Chem. 2001, 44, 3673.

24. Alves, C. N.; Pinheiro, J. C.; Camargo, A. J.; Ferreira, M. M. C.; Romero, R. A. F.; da Silva, A. B. F.; J. Mol. Struc-Theochem. 2001, 541, 81

25. De Oliveira, D. B.; Gáudio, A. C.; Quant. Struct-Act. Rel. 2001, 19, 599.

26. Borges, E. G.; Takahata, Y.; J. Mol. Struc-Theochem. 2001, 539, 245.

27. Pinto; M. F. S.; Romero, O. A. S.; Pinheiro, J. C.; J. Mol. Struc-Theochem. 2001, 539, 303

28. La-Scalea, M. A.; Chin, C. M.; Cruz, M. L.; Serrano, S. H. P.; Ferreira, E. I.; Bioelectrochemistry 2001, 53, 55

29. Andricopulo, A. D.; Yunes R. A.; Chem. Pharm. Bull. 2001, 49, 10

30. Krogh, R.; Yunes, R. A.; Andricopulo, A. D.; Farmaco 2000, 55, 730

31. Alves, C. N.; Pinheiro, J. C.; Camargo, A. J.; Ferreira, M. M. C.; da Silva, A. B. F.; J. Mol. Struc-Theochem. 2000, 530, 39

32. Montanari, M. L. C.; Beezer, A. E.; Montanari, C. A.; Pilo-Veloso, D,; J. Med. Chem. 2000, 43, 3448

33. Menezes, F. A. S.; Montanari, C. A.; Bruns, R. E.; J. Braz. Chem. Soc. 2000, 393

34. Amboni, R. D. D. C.; Junkes, B. D.; Yunes, R. A.; Heinzen, V. E. F.; J. Agric. Food Chem. 2000, 48, 3517.

35. Gaudio, A. C.; Richards, W. G.; Takahata, Y.; J. Mol. Graph. Model. 2000, 18,33 .

36. Montanari, M. L. C.; Cass, Q. B.; Montanari, C.A.; Chromatographia 2000, 51,722

37. Rodrigues, R. D.; Lopes, J. C. D.; Montanari, C. A.; Quant. Struct-Act. Rel. 2000, 19, 173 .

38. Leitão, A.; Montanari, C. A.; Donnici, C. L.; Quim. Nova 2000, 23, 178.

39. Figueiredo, L. J. O.; Antunes, O. A. C.; Int. J. Quantum Chem. 2000, 76, 744

40. Alves, C. N.; Pinheiro, J. C.; Camargo, A. J.; de Souza, A. J.; Carvalho, R. B.; da Silva, A. B. F.; J. Mol. Struc-Theochem. 1999, 491, 123.

41. Andricopulo, A. D.; Yunes, R. A.; Cechinel, V.; Nunes, R. J.; Frazer, J. W.; Cordes, E. H.; Pharmazie 1999, 54, 698.

42. Matos, M. S.; Gehlen, M. H.; Trsic, M.; Theochem-J. Mol. Struc. 1999, 488, 233.

43. Montanari, M. L. C.; Beezer, A. E.; Montanari, C. A.; Thermochim. Acta 1999, 333, 191.

44. Heinzen, V. E. F.; Cechinel, V.; Yunes, R. A.; Fármaco 1999, 54, 125

45. Magalhães, N. S. S.; Cavalcanti, S. C. D.; De Menezes, I. R. A.; Araújo, A. A. D.; De Oliveira, H. M.; Alves, A. J.; Eur. J. Med. Chem. 1999, 34, 83.

46. Woolfrey, J. R.; Rodrigues, C. R.; Alvim-Gaston, M.; Barreiro, E. J.; Cohen, F. E.; Avery, M. A.; Abstr. Pap. Am. Chem. 1999, S 217, U681.

47. Montanari, M. L. C.; Beezer, A. E.; Montanari, C. A.; Thermochim. Acta 1999, 328, 91 .

48. Dos Santos, H. F.; O'malley, P. J.; De Almeida, W. B.; Theor. Chem. Acc. 1998, 99, 301.

49. Albuquerque, M. G.; Hopfinger, A. J.; Barreiro, E. J.; de Alencastro, R. B.; J. Chem. Inf. Comp. Sci. 1998, 38, 925.

50. Montanari, M. L. C.; Montanari, C. A.; Veloso, D. P.; Beezer, A. E.; Mitchell, J. C.; Volpe, P. L. O.; Quant. Struct-Act. Rel. 1998, 17, 102.

51. Montanari, C. A.; Tute, M. S.; Quant. Struct-Act. Rel. 1997, 16, 480.

52. Costa, M. C. A.; Gaudio, A. C.; Takahata, Y.; Theochem-J. Mol. Struc. 1997, 394, 291.

53. do Amaral, A. T.; Oliveira, A. C.; Neidlein, R.; Gallacci, M.; Caprara, L.; Miyazaki, Y.; Eur. J. Med. Chem. 1997, 32, 433.

54. Vianna, J. F.; Aydos, M. C. R.; Siqueira, O. S.; Quim. Nova 1997, $20,125$.

55. Pires, J. M.; Floriano, W. B.; Gáudio, A. C.; Theochem-J. Mol. Struc. 1997, 389, 159.

56. Moreno, M. D. N.; Magalhães, N. S. S.; Cavalcanti, S. C. H.; Alves, A. J.; Ouim. Nova 1996, 19, 594.

57. Gaudio, A. C.; Quim. Nova 1996, 19, 278.

58. Menezes, C. M. S.; Kirchgatter, K.; Disanti, S. M. F.; Savalli, C.; Monteiro, F. G.; Paula, G. A.; Ferreira, E. I.; Abstr. Pap. Am. Chem. 1996, S 211, 189.
59. Montanari, C. A.; Quim. Nova 1995, 18, 56.

60. Smith, S.; Furay, V. J.; Layiwola, P. J.; Menezesfilho, J. A.; Chemosphere 1994, 28, 825 .

61. Richard, J. M.; Taillandier, G.; Ramos, M. N.; Galdino, S. L.; Pitta, I. D.; Pharmazie 1990, 45, 771.

62. Ramos, M. N.; Neto, B. D.; J. Comput. Chem. 1990, 11, 569.

63. Grynberg, N.; Gomes, R.; Shinzato, T.; Echevarria, A.; Miller, J.; Anticancer Research 1992, 12, 1025.

64. Reina-San-Martin, B.; Degrave, W.; Rougeot, C.; Cosson, A.; Chamond, N.; Cordeiro-Da-Silva, A.; Arala-Chaves, M.; Coutinho, A.; Minoprio, P.; Nat. Med. 2000, 6, 890 .

65. Fothergill-Gilmore, L. A.; Rigden, D. J.; Michels, P. A. M.; Phillips, S. E. V.; Biochem. Soc. T. 2000, 28, 186.

66. La-Scalea, M. A.; Serrano, S. H. P.; Gutz, I. G. R.; Quim. Nova 1999, 22, 417

67. Chin, C. M.; Ferreira, E. I.; Quim. Nova 1999, 22, 75.

68. Canduri, F.; Teodoro, L. G. V. L.; Lorenzi, C. C. B.; Gomes, R. A. S.; Fontes, M.R. M.; Arni, R. K.; de Azevedo, W. F.; Biochem. Mol. Biol. Int. 1998, 46, 355 .

69. Harvey, A. L.; Bradley, K. N.; Cochran, S. A.; Rowan, E. G.; Pratt, J. A.; Quillfeldt, J. A.; Jerusalinsky, D. A.; Toxicon. 1998, 36, 1635.

70. Montanari, C. A.; Trent, J. O.; Jenkins, T. C.; J. Braz. Chem. Soc. 1998, 9, 175

71. Costa-Campos, L.; Lara, D. R.; Nunes, D. S.; Elisabetsky E.; Pharmacol. Biochem. Be. 1998, 60, 133.

72. Grynberg, N.; Santos, A. C.; Echevarria, A.; Anti-Cancer Drugs 1997, 8, 88

73. Armelin, M. C. S.; Sasahara, R. M.; Flatschart, R.; Vedoy, C.; Braz. J. Med. Biol. Res. 1996, 29, 1751.

74. Fonteles, M. C.; Huang, L. C.; Larner, J.; Diabetologia 1996, 39, 731.

75. Lasserre, R.; Kessler, R.; Mem. I. Oswaldo Cruz 1992, 87, 249.

76. Mascarenhas, Y. P.; Stouten, P. F. W.; Beltran, J. R.; Laure, C. J.; Vriend, G.; Eur. Biophys. J. Biophy. 1992, 21, 199.

77. Deoliveira, M. M.; Mem. I. Oswaldo Cruz 1991, 86, 61.

78. Genestra, M.; Echevarria, A.; Cysne-Finkelstein, L.; Leon, L. L.; Arzneimittel-Forsch. 2001, 51, 920.

79. Moreira, L. S. A.; Pilo-Veloso, D.; Nelson, D. L.; Quim. Nova 2000, 23, 447.

\section{APÊNDICE}

\section{DIVISÃO DE QUÍMICA MEDICINAL}

\section{Número de filiados: 166 (atual)}

2000-2002

DIRETOR

VICE-DIRETOR:

Ricardo Bicca de Alencastro

TESOUREIRO

Carlos M. R. de Sant'Anna

1998-2000

DIRETOR

VICE-DIRETOR

Elisabeth Igne Ferreira

TESOUREIRO:

Carlos Alberto Montanari

Eliezer J. de L. Barreiro

Ricardo Bicca de Alencastro

1996-1998

DIRETOR:

VICE DIRETOR

Eliezer J. de L. Barreiro

TESOUREIRO:

Antonia Tavares do Amaral

Carlos Alberto Montanari

1994-1996

DIRETOR

VICE-DIRETOR:

Antonia Tavares do Amaral

TESOUREIRO:
Eliezer J. de L. Barreiro

Rozendo A. Yunes 